\title{
A LIQUID-TRIGGERED LIQUID MICROVALVE
}

\author{
Jessica Melin, Niclas Roxhed, Guillem Gimenez, Patrick Griss ${ }^{1}$, Wouter van der Wijngaart, Göran Stemme \\ Microsystem Technology, Department of Signals, Sensors, and Systems, Royal Institute of Technology, \\ Stockholm, Sweden
}

\begin{abstract}
This work introduces a novel surface tension and geometry based liquid-triggered liquid microvalve for on-chip liquid control. The simultaneous presence of two liquid plugs at the uncomplicated valve junction triggers the further movement of the liquids and overcomes the stop valve function of the device, thereby providing a precise means of timing liquid movement onchip. The structure was shown to successfully function and forms the basis for several novel and useful functions, including fluidic AND gates, contactless on-chip liquid sample control, timing of independent processes on the same microchip, bubble-free joining of liquids, all of which pose great challenges in the area of microfluidics.
\end{abstract}

\section{INTRODUCTION}

Micro-fluidic systems are of particular importance in the fields of biology, chemistry, and biomedicine due to a need for handling and controlling volumes in the range of nanolitres. Such systems open up many opportunities in application areas such as proteomics, genomics, drug delivery, high throughput screening, etc. Sample mixing and reaction of chemicals are common processes which require time control of the liquids on-chip [1]. A functional component is required if two sample volumes are to be brought into contact at a specific location on-chip to ensure that both liquids arrive simultaneously. When two liquids meet at an intersection, an air pocket becomes trapped if the liquids do not reach the junction simultaneously. Evacuating two channels through a common outlet [2] or joining two channels at the inlet of a reaction/mixing chamber thus introduces an air-pocket problem as is demonstrated in Figure 1. Precisely timing the initial contact of two liquids at the inlet of a reaction/mixing chamber is particularly important in microsystems where mixing time is of the same order of magnitude as the reaction's time constant [3].

\footnotetext{
${ }^{1}$ Patrick Griss' current address is Roche Diagnostics Microtechnology Center, 6343 Rotkreutz, Switzerland.
}

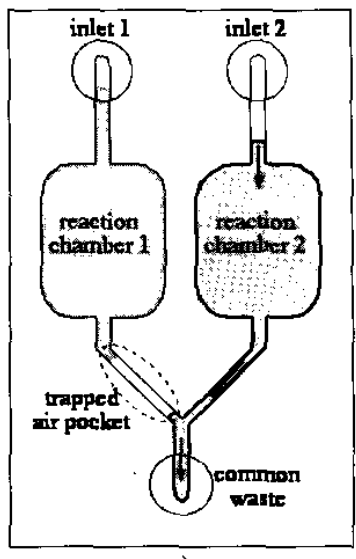

a)

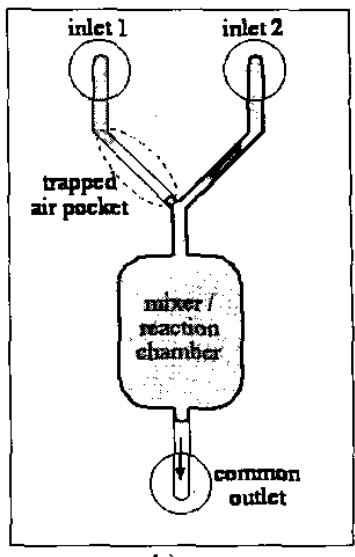

b)
Figure 1. a) Schematic showing problem of trapped air at the inlet of a mixer, and b) a similar problem where two liquids are directed to the same outlet channel at different moments in time. Both problems can be circumvented by using the novel $\mathrm{Y}$-junction.

Active valving is one solution to the challenges described above. However, in microsystems, valves often carry challenges such as complex fabrication and packaging, moving parts, bulky external actuation principles, leakage, and discrimination towards liquids commonly used in biotechnology. Passive mechanism where patterned hydrophobic areas act as stop valves used for liquid control have been presented [2]. Geometrically defined and capillarily driven stop valves with no moving parts have also been presented as injectors where the stop function is overcome by electrical heating to create a pressure difference [4]. We present a geometrically defined and uncomplicated functional structure for controlling two or more liquids on-chip where the stop function is overcome, i.e. timing of liquid movement is achieved simply by the presence of the other liquid samples at the device junction.

\section{DEVICE DESIGN}

The basic microvalve consists of a $Y$ junction with two inlet ports and one outlet port as can be seen in Figure 2. The key to its functionality lies in the junction geometry. The junction acts as a geometrical stop-valve when liquid from either port 1 or port 2 reaches this point [4]. However, the junction allows liquid to flow 
through to the outlet when liquid from both inlet ports are present at the junction. Thus, liquid from one inlet port reaching the junction, either by capillary forces or externally driven, waits for liquid from the other inlet port to reach the junction before moving through the outlet (i.e. the movement of the first liquid is triggered by the presence of the second liquid at the junction). Figure 3 shows a schematic and photographs of the consecutive steps in the functionality of the structure.

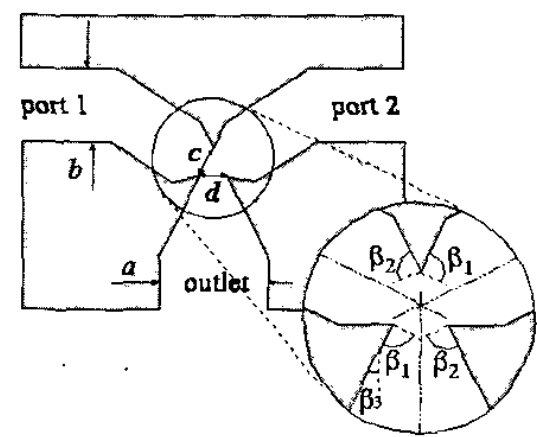

Figure 2. Schematic of the novel $\mathrm{Y}$-junction. In the realized structures $a=100 \mu \mathrm{m}, b=50 \mu \mathrm{m}, \mathrm{c}=11 \mu \mathrm{m}, d=$ $4 \mu \mathrm{m}, \beta_{3}=15$, and $\beta_{1}=\beta_{2}>85$. a)
Schematic Functionality of Device

b)

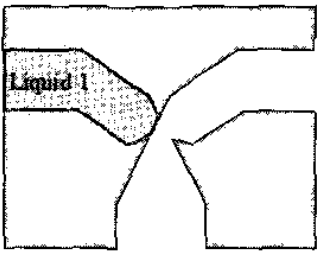

c)

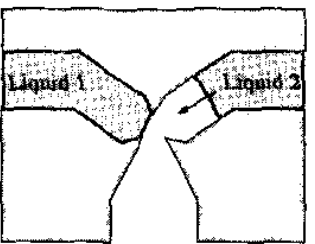

i)

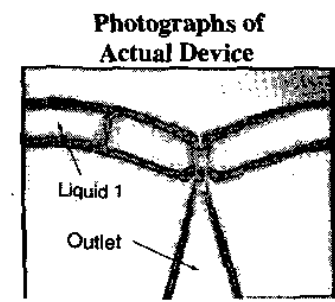

ii)

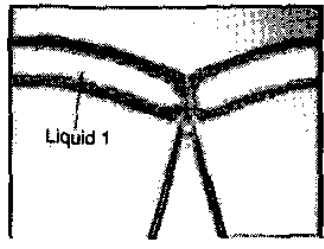

iii)

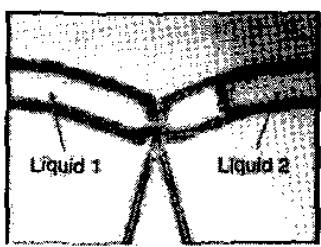

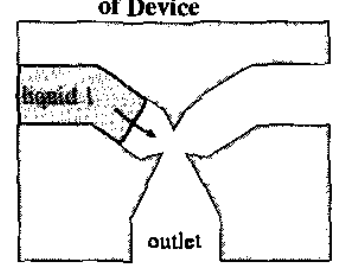

d)

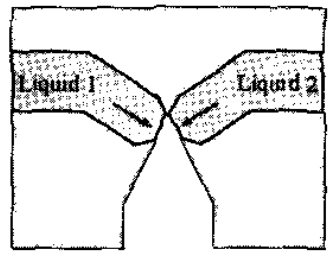

e)

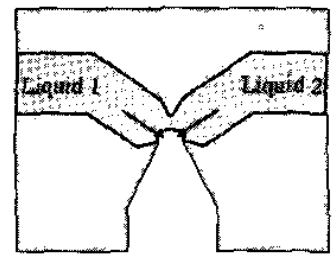

iv)

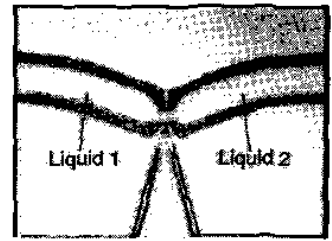

v)

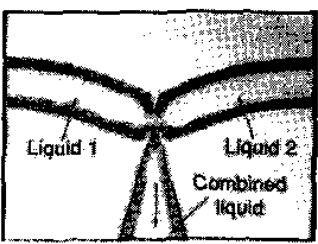

Figure 3. a),i) Liquid 1 enters inlet port 1, b),ii) due to the geometry of the junction, liquid 1 stops at the junction, c),iii) liquid 2 enters inlet port 2, d), e) and iv) liquid 1 and 2 make contact at the junction and proceed vi),f) liquid 1 and 2 proceed to fill the outlet, capillarily or pressure driven (depending on $\beta_{3}$ and the contact angle $\theta_{\mathrm{s}}$ ).

\section{THEORY}

The total surface energy in a fluidic channel system containing different solid surfaces $s$, and containing one liquid phase and one gas phase can be expressed as

$U=A_{\mathrm{lg}} \gamma_{\mathrm{Ig}}+\sum_{s}\left(A_{s l} \gamma_{s l}+A_{s g} \gamma_{s g}\right)$

where the indices $s, l$ and $g$ respectively indicate the solid phase, the liquid phase and the gas phase, $A_{i j}$ indicates the interface area between the different phases $i$ and $j, \gamma_{i j}$ indicates the surface energy of the interfaces and the summation adds over all solid phase interfaces. Taking into account Young's equation for a solid-liquid-gas interface line

$\gamma_{s g}=\gamma_{s l}+\gamma_{\mathrm{lg}} \cos \theta_{s}$

and the fact that the sum of all solid phase areas $A_{s l}$ and $A_{s g}$ is constant, one can express the internal liquid pressure as

$\left.P_{1}=-\frac{d U}{d V_{l}}=\gamma_{\mathrm{lg}} \cdot \quad\left(\cos \theta_{s} \frac{d A_{s l}}{d V_{l}}\right)-\frac{d A_{\mathrm{lg}}}{d V_{l}}\right)$ 
where $V_{l}$ is the liquid volume in the channel (freely adapted from [4]). If $P_{l}$ is positive, liquid will be drawn into the channel, i.e. capillary filling occurs. If $P_{l}$ becomes negative, e.g. in an abruptly expanding channel or when using hydrophobic surfaces, the liquid will be repulsed from the channel.

Assuming a cylindrical liquid front, as schematically shown in Figure 4 , the pressure $\left(P_{I}\right)$ required for the liquid to travel through a $3 \mathrm{D}$ tapered channel where the walls and floor of the channel are silicon and the roof of the channel is PDMS (polydimethylsilasane) can be defined as

$$
\left.P_{1}=\gamma_{\mathrm{lg}} \cdot \cos \theta_{S i} \frac{d A_{S i}}{d V_{l}}+\cos \theta_{P D M S} \frac{d A_{P D M S}}{d V_{l}}-\frac{d A_{\mathrm{lg}}}{d V_{l}}\right)
$$

in which $A_{S i}$ and $A_{P D M S}$ are the respective wetted silicon and PDMS areas and where $\alpha, \beta$, and $R$ are defined in Figure 4 and $h$ is the height of the channel. Assuming a cylindrical liquid front, one can express

$$
\begin{aligned}
& d A_{S i l}=2 h d R+c 2 R d R \\
& d A_{P D M S l}=c 2 R d R \\
& d V_{l}=c h 2 R d R
\end{aligned}
$$

where

$c(\alpha, \beta)=\sin \beta \cos \beta-\left(\frac{\sin \beta}{\sin \alpha}\right)^{2}(\alpha-\sin \alpha \cos \alpha)$

and $\alpha=\frac{\pi}{2}-\beta-\theta_{s}$.

The pressure $P_{l}$ at the channel neck with width $w=R \cos \beta$ can than be expressed as

$$
\left.P_{i}=\frac{\gamma_{l a}}{R h c} \cdot(h+R c) \cos \theta_{S i}+R c \cos \theta_{P D M S}-\frac{h \alpha \sin \beta}{\sin (\alpha)}\right)
$$

This pressure is negative for large angles of $\beta$, i.e. the geometry functions as a stop valve for the liquid. Equation (10) allows the pressure barrier for liquid reaching the junction via only one port to be calculated, as well as the pressure barrier occurring at large values of $\beta_{3}$ for liquids proceeding from the junction towards the outlet port.

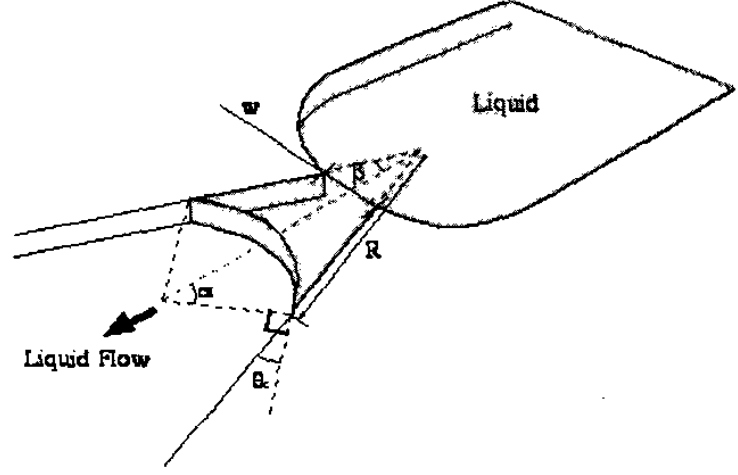

Figure 4. Schematic of liquid passing through a tapered channel.

\section{FABRICATION}

The device was fabricated by patterning a silicon wafer using photoresist, etching the pattern a depth of $50 \mu \mathrm{m}$ using deep reactive ion etching and subsequently removing the mask layer and cleaning the wafer. The wafer was diced into individual chips and packaged by covering the top surface with a layer of PDMS, thereby sealing the channel system. Holes were cut through the PDMS with a sharp steel tube at the inlet and outlet ports and syringes inserted and fixated with epoxy for testing purposes. Figure 5 shows a schematic of the fabricated device. The outlet channel width, $a$, was $100 \mu \mathrm{m}$, inlet channel width, $b$, was $50 \mu \mathrm{m}$, while the inlet junction width, $c$, was $11 \mu \mathrm{m}$ and outlet junction width, $d$, was $4 \mu \mathrm{m}$. The outlet taper angle, $\beta_{3}$ was 15 and $\beta_{1}$ and $\beta_{2}$ as defined in Figure 2 are both greater than 85 .

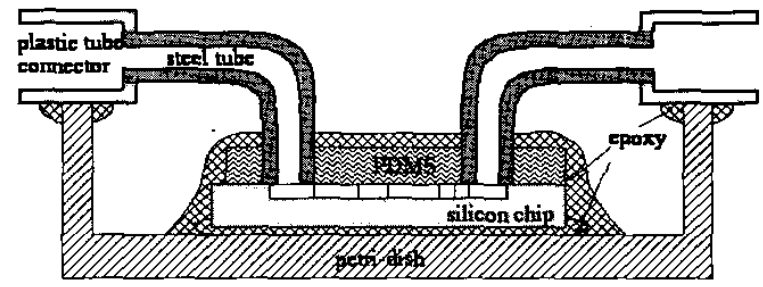

Figure 5. Cross-section schematic of packaged device used for testing.

\section{EXPERIMENTAL RESULTS}

To test the functionality of the device, deionized coloured water was entered into the inlet ports. Figure 3 shows photographs of the step-by-step functionality of the device which correspond well to the theoretical.

The measured pressure required to overcome the stop valve function (i.e. for liquid to overcome angle $\beta_{1}$ ) for water at a single inlet of the $Y$ junction was $7.3 \mathrm{kPa}$ (i.e. for liquid to flow from a single 
inlet to the outlet). This pressure is the upper limit for pressure driven flow in the measured junction. Note that the $\mathrm{Y}$-junction must be dried before reuse.

\section{DEVICE APPLICATIONS}

The liquid-triggered liquid microvalve is the basis for a variety of devices which may solve a number of microfluidic challenges and can be integrated with microfluidic systems on-chip. The junction allows liquid channels or reaction chambers to be evacuated simultaneously or joined without trapping gas bubbles as described in the introduction. Figure 6a) shows another application where liquid in one inlet port triggers the liquid in another inlet port to move to its respective outlet. The design can be expanded as in Figure 6b) to include a waste outlet to minimize the risk of any crosscontaminated liquid proceeding through the outlet channels.

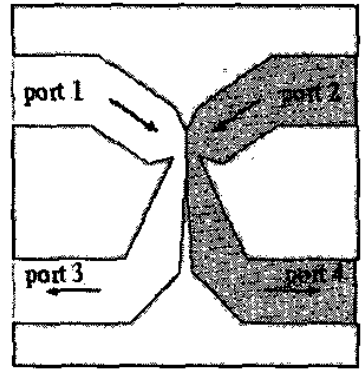

a)

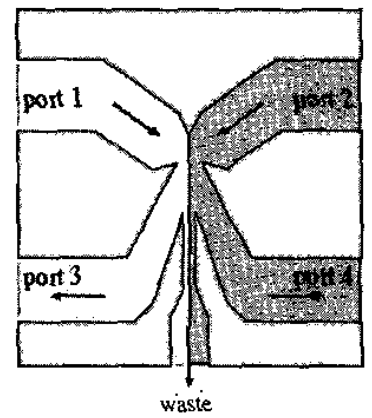

b)
Figure 6. Schematic of a) a Y-junction used to trigger independent processes on the same chip (port 3 and port 4) and b) an extension of the same application with a waste outlet.

If the junction is designed so that the liquids only proceed if a negative pressure (suction) is applied at the outlet (when $\beta_{3}$ is large), a new functionality arises. A defined location for two liquids to react and $\mathrm{mix}$ by diffusion has been created and can be monitored, as illustrated in Figure 7.

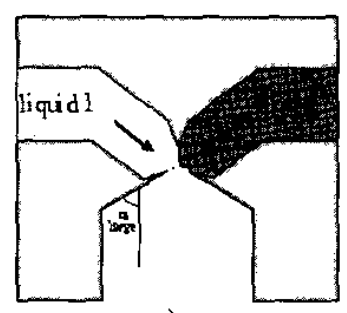

a)

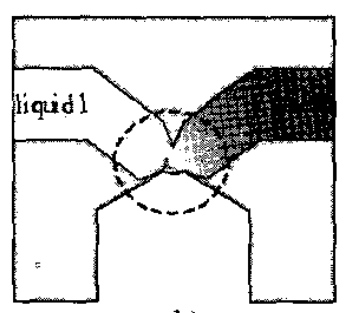

b)
Figure 7. Schematic showing Y-junction as a defined location to bring two liquids in contact for reacting, mixing or monitoring. a) liquids arrige and stop at the junction, b) liquids begin to diffuse into each other.
The AND gate principle can also be expanded to n-to-1 AND gates as shown in Figure 8. The valve does not allow any of the incoming liquids to proceed unless all liquids have arrived at the junction. The liquid-triggered microvalve lends a solution to several common challenges in terms of controlling movement and interaction of liquids in microsystems.

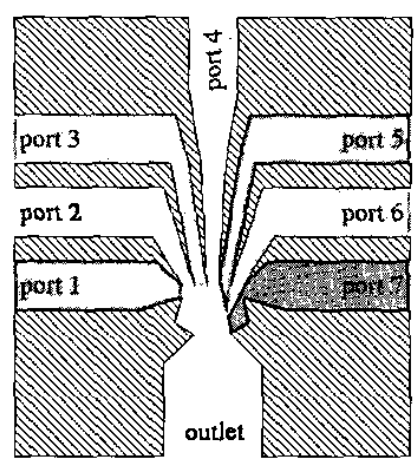

Figure 8. Example of expanding the $\mathrm{Y}$-junction to form an n-to-1 AND gate. Liquids proceed to the outlet only when liquids from all inlet ports have reached the junction.

\section{CONCLUSIONS}

A novel, uncomplicated, geometrically based functional component has been presented as a means of controlling small liquid samples on-chip. The stop valve function of the component is only overcome when all liquids involved in the subsequent on-chip process, reaction, etc. arrive and make contact at the device junction. The device functionality was successfully tested. This liquid-triggered liquid microvalve can be the basis for solving various challenges in terms of timing and controlling small liquid samples in the field of biotechnology, drug delivery, etc.

\section{REFERENCES}

1.Micromachined Transducers Sourcebook, Kovacs, G.T.A., WCB McGraw Hill, New York, pg 815, 1998.

2."Micromachined filter-chamber array with passive valves for biochemical assays on beads," $H$. Andersson, W. van der Wijngaart, G. Stemme, Electrophoresis, 22, 249-257, 2001.

3."Microstructure for efficient continuous flow mixing," F.G. Bessoth, A.J. de Mello, A. Manz, Anal. Commun., 36, 213-215, 1999.

4."Microfabricated capillary-driven stop valve and sample injector," P.F. Man, C.H. Mastrangelo, M.A. Burns, D.T. Burke, MEMS Workshop, 45-50, 1998. 